\title{
Scalar Product in the Space of Waveguide Modes of an Open Planar Waveguide
}

\author{
Sevastianov A.L. ${ }^{1, a}$, Sevastianov L.A. ${ }^{1,2, b}$, Tiutiunnik A.A. ${ }^{1, c}$, and Nikolaev N.E. ${ }^{1, d}$ \\ ${ }^{1}$ Peoples' Friendship University of Russia, Miklukho-Maklaya str. 6, Moscow, 117198, Russia \\ 2 Joint Institute for Nuclear Research, Joliot-Curie 6, Dubna, Moscow region, 141980, Russia
}

\begin{abstract}
To implement the method of adiabatic waveguide modes for modeling the propagation of polarized monochromatic electromagnetic radiation in irregular integrated optics structures it is necessary to expand the desired solution in basic adiabatic waveguide modes. This expansion requires the use of the scalar product in the space of waveguide vector fields of integrated optics waveguide. This work solves the first stage of this problem - the construction of the scalar product in the space of vector solutions of the eigenmode problem (classical and generalized) waveguide modes of an open planar waveguide. In constructing the mentioned sesquilinear form, we used the Lorentz reciprocity principle of waveguide modes and tensor form of the Ostrogradsky-Gauss theorem.
\end{abstract}

\section{Introduction}

To describe the amplitude and phase matching on real (and / or imagined) interfaces of media (and/ /or regions) of the electromagnetic radiation propagating through these boundaries it is required to use an additional (to Maxwell equations) relation, similar to the law of energy conservation for the electromagnetic field, or the Lorentz lemma on reciprocity. The relations of this kind are also the boundary conditions of equality of the tangential components of the electromagnetic field on the real interface between two media through which the described electromagnetic radiation propagates.

Assume that there exists a first group of external currents $\vec{j}_{1}$, creating the electromagnetic (monochromatic) field $\left(\begin{array}{l}\vec{E}_{1} \\ \vec{H}_{1}\end{array}\right)$. Also assume that there exists a second group of external currents $\vec{j}_{2}$, creating the electromagnetic (monochromatic) field $\left(\begin{array}{l}\vec{E}_{2} \\ \vec{H}_{2}\end{array}\right)$. Then the relation, called Lorentz lemma on reciprocity in differential form is true [4]:

$$
\operatorname{div}\left[\vec{E}_{2} \times \vec{H}_{1}\right]-\operatorname{div}\left[\vec{E}_{1} \times \vec{H}_{2}\right]=\left(\vec{j}_{2 e}, \vec{E}_{1}\right)+\left(\vec{j}_{1 m}, \vec{H}_{2}\right)-\left(\vec{j}_{1 e}, \vec{E}_{2}\right)-\left(\vec{j}_{2 m}, \vec{H}_{1}\right),
$$

\footnotetext{
ae-mail: alsevastyanov@gmail.com

be-mail: leonid.sevast@gmail.com

ce-mail: nastya.tyutyunnik@gmail.com

de-mail: nkoalan@gmail.com
}

This is an Open Access article distributed under the terms of the Creative Commons Attribution License 4.0, which permits

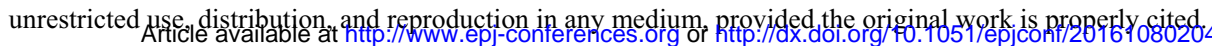


where the currents $\vec{j}_{1 e}$ and $\vec{j}_{2 e}$ create the fields $\vec{E}_{1}$ and $\vec{E}_{2}$, while $\vec{j}_{1 m}$ and $\vec{j}_{2 m}$ create the $\vec{H}_{1}$ and $\vec{H}_{2}$.

When the Lorentz lemma is applied to the area $\Omega$ filled with electromagnetic radiation generated by external currents which are concentrated outside $\Omega$, the relation (1) takes the following form:

$$
\operatorname{div}\left[\vec{E}_{2} \times \vec{H}_{1}\right]-\operatorname{div}\left[\vec{E}_{1} \times \vec{H}_{2}\right]=0 .
$$

Under the waveguide propagation of a monochromatic polarized electromagnetic radiation (optical range) in regular waveguides, the expressions of the electromagnetic field can be factored as:

$$
\left(\begin{array}{l}
\vec{E} \\
\vec{H}
\end{array}\right)_{ \pm}(\vec{r}, t, \beta)=\left(\begin{array}{l}
\vec{E} \\
\vec{H}
\end{array}\right)_{ \pm}(x, y, \beta) \exp \left\{i \omega t \mp i k_{0} \beta z\right\} .
$$

The use of the Lorentz lemma on reciprocity to the waveguide propagation of Maxwell optical radiation leads to the factorization of (2) into the sum of two terms involving the transverse electromagnetic field distributions and the exponential factor respectively. In the case of real $\varepsilon$ and $\mu$, the second contribution to the Lorentz lemma vanishes identically, while if $\operatorname{Im} \varepsilon \ll \operatorname{Re} \varepsilon$, it is negligibly small. The remaining first part of the sum, renamed as "conservation law", can be called "truncated Lorentz lemma" for the waveguide propagation of the electromagnetic radiation.

An independent derivation of the "truncated Lorentz lemma" was given in [3] for fiber-optics waveguides. In [5] the expression of "truncated Lorentz lemma" was suggested for planar regular waveguides without derivation.

The derivation of the "truncated Lorentz lemma" for waveguide modes of planar regular waveguides is a useful preliminary step in the derivation of the Lorentz lemma and the "truncated Lorentz lemma" for adiabatic waveguide modes of integrated optics waveguides. The latter relation is necessary for the derivation of the completeness of adiabatic waveguide modes of integrated optics waveguides, based on the matrix formulation of the scalar product of four-component expressions through the tangential components of the electromagnetic field of adiabatic waveguide modes [2].

\section{Adiabatic waveguide modes of smoothly irregular integrated optics waveguide}

If a solution is sought in the form

$$
\left(\begin{array}{l}
\vec{E} \\
\vec{H}
\end{array}\right)(x, y, z, t)=\left(\begin{array}{l}
\vec{E} \\
\vec{H}
\end{array}\right)(x ; y, z) \frac{\exp \{i \omega t-i \varphi(y, z)\}}{\sqrt{\beta(y, z)}},
$$

where $\beta^{2}=\beta_{y}^{2}+\beta_{z}^{2}$ and $\beta_{y}=\frac{\partial \varphi}{\partial y}, \beta_{z}=\frac{\partial \varphi}{\partial z}$, then the following relations are satisfied $\frac{\partial \Psi}{\partial z}=p_{z} \Psi, \frac{\partial \Psi}{\partial y}=$ $p_{y} \Psi$, where $p_{z}=-i \frac{d \varphi}{d z}-\frac{1}{\beta} \frac{d \beta}{d z}, p_{y}=-i \frac{d \varphi}{d y}-\frac{1}{\beta} \frac{d \beta}{d y}$. Substituting (4) into the Maxwell equations and taking into account these two relations, we obtain the following relations for the first derivatives of the field components

$$
\begin{gathered}
\frac{\partial H_{z}}{\partial x}=-\frac{1}{i k_{0} \mu}\left(\left(\frac{\partial p_{y}}{\partial z}+p_{y} p_{z}\right) E_{z}-\left(\frac{\partial p_{z}}{\partial z}+p_{z}^{2}+k_{0}^{2} \varepsilon \mu\right) E_{y}\right), \\
\frac{\partial H_{y}}{\partial x}=\frac{1}{i k_{0} \mu}\left(\left(\frac{\partial p_{z}}{\partial y}+p_{y} p_{z}\right) E_{y}-\left(\frac{\partial p_{y}}{\partial y}+p_{y}^{2}+k_{0}^{2} \varepsilon \mu\right) E_{z}\right), \\
\frac{\partial E_{z}}{\partial x}=\frac{1}{i k_{0} \varepsilon}\left(\left(\frac{\partial p_{y}}{\partial z}+p_{y} p_{z}\right) H_{z}-\left(\frac{\partial p_{z}}{\partial z}+p_{z}^{2}+k_{0}^{2} \varepsilon \mu\right) H_{y}\right),
\end{gathered}
$$




$$
\frac{\partial E_{y}}{\partial x}=\frac{1}{i k_{0} \varepsilon}\left(\left(\frac{\partial p_{z}}{\partial y}+p_{y} p_{z}\right) H_{y}-\left(\frac{\partial p_{y}}{\partial y}+p_{y}^{2}+k_{0}^{2} \varepsilon \mu\right) H_{z}\right) .
$$

In (7) and (8), the terms proportional to $\frac{\partial}{\partial z}\left(\frac{1}{\varepsilon}\right)$ and to $\frac{\partial}{\partial y}\left(\frac{1}{\varepsilon}\right)$ have been neglected. These terms are small as compared to other values in the gradient smoothly irregular waveguides and vanish identically in the multilayer smoothly irregular waveguides [6].

Further, we substitute into the Maxwell equations the solution of the adiabatic waveguide modes in the form (4), which contains (initially) only guided waveguide modes corresponding to the discrete spectrum,

$$
u(x, y, z, t)=\sum_{j} C_{j}(y, z) \Psi_{j}(x, y, z, t)
$$

After a series of transformations and after taking into account (5)-(8), we obtain the following relations in matrix-vector form for the solutions (9):

$$
\begin{gathered}
\sum_{j}\left(\begin{array}{cc}
\frac{\partial^{2} C_{j}}{\partial z^{2}}+2 p_{z_{j}} \frac{\partial C_{j}}{\partial z} & -\frac{\partial^{2} C_{j}}{\partial z \partial y}-p_{y_{j}} \frac{\partial C_{j}}{\partial z}-p_{z j} \frac{\partial C_{j}}{\partial y} \\
-\frac{\partial^{2} C_{j}}{\partial y \partial z}-p_{z_{j}} \frac{\partial C_{j}}{\partial y}-p_{y_{j}} \frac{\partial C_{j}}{\partial z} & \frac{\partial^{2} C_{j}}{\partial y^{2}}+2 p_{y_{j}} \frac{\partial C_{j}}{\partial y}
\end{array}\right)\left(\begin{array}{l}
E_{y_{j}} \\
E_{z_{j}}
\end{array}\right)=\left(\begin{array}{l}
0 \\
0
\end{array}\right), \\
\sum_{j}\left(\begin{array}{cc}
\frac{\partial^{2} C_{j}}{\partial z^{2}}+2 p_{z_{j}} \frac{\partial C_{j}}{\partial z} & -\frac{\partial^{2} C_{j}}{\partial z \partial y}-p_{y_{j}} \frac{\partial C_{j}}{\partial z}-p_{z j} \frac{\partial C_{j}}{\partial y} \\
-\frac{\partial^{2} C_{j}}{\partial y \partial z}-p_{z j} \frac{\partial C_{j}}{\partial y}-p_{y_{j}} \frac{\partial C_{j}}{\partial z} & \frac{\partial^{2} C_{j}}{\partial y^{2}}+2 p_{y_{j}} \frac{\partial C_{j}}{\partial y}
\end{array}\right)\left(\begin{array}{l}
H_{y_{j}} \\
H_{z_{j}}
\end{array}\right)=\left(\begin{array}{l}
0 \\
0
\end{array}\right),
\end{gathered}
$$

which is equivalent to $\mathbf{M}(D C) \boldsymbol{\Psi}=\mathbf{0}$ with the $4 \times 4$ matrix $\mathbf{M}$ shown in (10)-(11), and the vector

$$
\boldsymbol{\Psi}=\left(\begin{array}{c}
E_{y} \\
E_{z} \\
H_{y} \\
H_{z}
\end{array}\right) .
$$

The scalar multiplication of this system with the corresponding coordinate functions, results in a system of partial differential equations of the second order with respect to the unknown coefficient functions $C_{j}(y, z)$. However, working with four-dimensional vectors and matrices requires a matrix scalar product. Further we proceed to the construction of this scalar product.

\section{Truncated Lorentz lemma}

We consider the planar regular isotropic optical (dielectric) waveguide.

In the absence of external charges and currents (inside the domain of interest) the optical electromagnetic radiation is described by the equations:

$$
\operatorname{rot} \vec{H}=\frac{\varepsilon}{c} \frac{\partial \vec{E}}{\partial t}, \quad \operatorname{rot} \vec{E}=-\frac{\mu}{c} \frac{\partial \vec{H}}{\partial t}
$$

with the boundary conditions for the tangential components $\vec{E}^{\tau}, \vec{H}^{\tau}$ at the borders $x=a_{1}$ and $x=a_{2}$

$$
\left.\vec{E}^{\tau}\right|_{x=a_{\alpha}-0}=\left.\vec{E}^{\tau}\right|_{x=a_{\alpha}+0},\left.\quad \vec{H}^{\tau}\right|_{x=a_{\alpha}-0}=\left.\vec{H}^{\tau}\right|_{x=a_{\alpha}+0}, \quad(\alpha=1,2)
$$

and the conditions at infinity for the electric $(\vec{E})$ and magnetic $(\vec{H})$ fields

$$
\|\vec{E}\|_{x \rightarrow \pm \infty} \leq C_{E}, \quad\|\vec{H}\|_{x \rightarrow \pm \infty} \leq C_{H}
$$


As shown in [6] the particular solutions of the problem (13)-(15) form two complete systems of solutions (TE- and TM- modes) for two Sturm-Liouville problems associated to it. In the Cartesian system of coordinates, the TE- and TM-modes are written as:

$$
\left(\begin{array}{c}
0 \\
E_{y} \\
0 \\
H_{x} \\
0 \\
H_{z}
\end{array}\right)(x) \exp \left\{i \omega t-i k_{0} \beta z\right\}, \quad\left(\begin{array}{c}
E_{x} \\
0 \\
E_{z} \\
0 \\
H_{y} \\
0
\end{array}\right)(x) \exp \left\{i \omega t-i k_{0} \beta z\right\} .
$$

The relations (16), under the condition $\partial / \partial y\left(\begin{array}{l}\vec{E} \\ \vec{H}\end{array}\right)=\left(\begin{array}{l}\overrightarrow{0} \\ \overrightarrow{0}\end{array}\right)$ are solutions of Sturm-Liouville problems with mixed spectrum.

For solutions of the form (16), the system of Maxwell equations (13) in Cartesian coordinates splits into two independent subsystems: for the TE-modes:

$$
\frac{d E_{y}}{d x}=-i k_{0} \mu H_{z}, \quad \frac{d H_{z}}{d x}=-i k_{0} \varepsilon E_{y}-i k_{0} \beta H_{x}, \quad-i k_{0} \mu H_{x}=i k_{0} \beta E_{y},
$$

and for the TM-modes:

$$
\frac{d H_{y}}{d x}=i k_{0} \varepsilon E_{z}, \quad \frac{d E_{z}}{d x}=i k_{0} \mu H_{y}-i k_{0} \beta E_{x}, \quad i k_{0} \varepsilon E_{x}=i k_{0} \beta H_{y} .
$$

Moreover, the positive values of a discrete parameter correspond to the "direct" waveguide modes propagating from left to right in the direction of increasing coordinate $z$, whereas the "reverse" waveguide modes propagating from right to left in the direction of decreasing coordinate $z$ can be described by negative values of the discrete spectral parameter [7]. The systems of equations corresponding to the reverse waveguide modes are: for the TE-modes

$$
\frac{d E_{y}}{d x}=-i k_{0} \mu H_{z}, \quad \frac{d H_{z}}{d x}=-i k_{0} \varepsilon E_{y}+i k_{0} \beta H_{x}, \quad-i k_{0} \mu H_{x}=-i k_{0} \beta E_{y},
$$

and for the TM- modes:

$$
\frac{d H_{y}}{d x}=i k_{0} \varepsilon E_{z}, \quad \frac{d E_{z}}{d x}=i k_{0} \mu H_{y}+i k_{0} \beta E_{x}, \quad i k_{0} \varepsilon E_{x}=-i k_{0} \beta H_{y} .
$$

Just as it was done in [3] for the waveguide modes of a fiber-optics waveguide, we apply the Lorentz lemma to the waveguide modes of planar waveguide. We split the divergence operator into longitudinal and transverse parts, and to the transverse part (the truncated Lorentz lemma) we apply the Ostrogradsky-Gauss theorem. The obtained result coincides with that of [5], namely, the scalar product in the class of vector fields (solutions of the Maxwell equations) is given by

$$
\left\langle\left(\begin{array}{l}
\vec{E} \\
\vec{H}
\end{array}\right)(\beta),\left(\begin{array}{l}
\vec{E} \\
\vec{H}
\end{array}\right)\left(\beta^{\prime}\right)\right\rangle=\int d x\left(\left[\vec{E}^{+}(\vec{r}, \beta) \times \vec{H}^{-}\left(\vec{r},-\beta^{\prime}\right)\right], \vec{e}_{z}\right),
$$

where the superscripts " \pm " indicate forward and reverse waveguide modes.

A direct verification shows that

$$
\left\langle\left(\begin{array}{l}
\vec{E} \\
\vec{H}
\end{array}\right)_{\mathrm{TE}}(\beta),\left(\begin{array}{l}
\vec{E} \\
\vec{H}
\end{array}\right)_{\mathrm{TE}}\left(\beta^{\prime}\right)\right\rangle=\frac{\beta^{\prime}}{\mu} \exp \left\{i k_{0}\left(\beta-\beta^{\prime}\right) z\right\} \int d x E_{y}^{\mathrm{TE}}(x, \beta) E_{y}^{\mathrm{TE}}\left(x,-\beta^{\prime}\right) .
$$


Similar verification for TM- modes yields

$$
\left\langle\left(\begin{array}{l}
\vec{E} \\
\vec{H}
\end{array}\right)_{\mathrm{TM}}(\beta),\left(\begin{array}{l}
\vec{E} \\
\vec{H}
\end{array}\right)_{\mathrm{TM}}\left(\beta^{\prime}\right)\right\rangle=\frac{\beta}{\varepsilon} \exp \left\{i k_{0}\left(\beta-\beta^{\prime}\right) z\right\} \int d x H_{y}^{\mathrm{TM}}(x, \beta) H_{y}^{\mathrm{TM}}\left(x,-\beta^{\prime}\right) .
$$

The mutual scalar products of the vector electromagnetic fields of the TE- and TM-waveguide modes equal zero.

\section{Full Lorentz lemma}

Our main problem is to describe the electromagnetic fields of the TE- and TM-waveguide modes through the four tangential components satisfying the system of linear first order ODEs,

$$
\begin{array}{ll}
\frac{d E_{y}}{d x}=-i k_{0} \mu H_{z}, & \frac{d H_{z}}{d x}=-i k_{0}\left(\varepsilon-\frac{\beta^{2}}{\mu}\right) E_{y}, \\
\frac{d H_{y}}{d x}=i k_{0} \varepsilon E_{z}, & \frac{d E_{z}}{d x}=i k_{0}\left(\mu-\frac{\beta^{2}}{\varepsilon}\right) H_{y} .
\end{array}
$$

The system (24) is valid for both direct and reverse waveguide modes, the obvious correspondence to the systems (17)-(20).

In order to write the scalar product through four tangential components satisfying the system of linear ordinary differential equations (24), we use the second and fourth equations to transform the expressions (22) and (23) to the form:

$$
\left\langle\left(\begin{array}{l}
\vec{E} \\
\vec{H}
\end{array}\right)_{\mathrm{TE}}(\beta),\left(\begin{array}{l}
\vec{E} \\
\vec{H}
\end{array}\right)_{\mathrm{TE}}\left(\beta^{\prime}\right)\right\rangle=\frac{i \beta^{\prime}}{k_{0}\left(\varepsilon \mu-\beta^{2}\right) \mu} \exp \left\{i k_{0}\left(\beta-\beta^{\prime}\right) z\right\} \int d x \frac{d H_{z}^{\mathrm{TE}}}{d x}(x, \beta) E_{y}^{\mathrm{TE}}\left(x,-\beta^{\prime}\right)
$$

and

$$
\left\langle\left(\begin{array}{l}
\vec{E} \\
\vec{H}
\end{array}\right)_{\mathrm{TM}}(\beta),\left(\begin{array}{l}
\vec{E} \\
\vec{H}
\end{array}\right)_{\mathrm{TM}}\left(\beta^{\prime}\right)\right\rangle=-\frac{i \beta}{k_{0}\left(\varepsilon \mu-\beta^{\prime 2}\right)} \exp \left\{i k_{0}\left(\beta-\beta^{\prime}\right) z\right\} \int d x H_{y}^{\mathrm{TM}}(x, \beta) \frac{d E_{z}^{\mathrm{TM}}}{d x}\left(x,-\beta^{\prime}\right) .
$$

To complete the argument, we make all the calculations directly using the full Lorentz lemma. In accordance with the main principle forming the basis of the full Lorentz lemma, the direct waveguide modes of the form (16) satisfying the systems (17) and (18) are generated by sources of the electromagnetic field to the left of the considered area of the waveguide and are marked by the index 1 . The reverse waveguide modes have the same form as (16) except the sign before $i k_{0} \beta z$. They satisfy the systems (19), (20), are generated by sources of the electromagnetic field to the right of the considered area of the waveguide, and are marked by the index 2. For this pair of electromagnetic radiation fields, the Lorenz lemma (2) is true. Direct calculation of the divergence leads to expressions identical to (21) and (22), (23) for the scalar products. Namely,

$$
\begin{aligned}
& \operatorname{div}\left[\vec{E}_{1}^{\mathrm{TE}}\left(\beta_{1}\right) \times \vec{H}_{2}^{\mathrm{TE}}\left(\beta_{2}\right)\right]=\frac{d}{d x}\left\{E_{y}^{\mathrm{TE}}\left(x, \beta_{1}\right) H_{z}^{\mathrm{TE}}\left(x,-\beta_{2}\right)\right\} \exp \left\{-i k_{0}\left(\beta_{1}-\beta_{2}\right) z\right\}- \\
& -i k_{0}\left(\beta_{1}-\beta_{2}\right) E_{y}^{\mathrm{TE}}\left(x, \beta_{1}\right) H_{x}^{\mathrm{TE}}\left(x,-\beta_{2}\right) \exp \left\{-i k_{0}\left(\beta_{1}-\beta_{2}\right) z\right\} \\
& \operatorname{div}\left[\vec{E}_{2}^{\mathrm{TE}}\left(\beta_{2}\right) \times \vec{H}_{1}^{\mathrm{TE}}\left(\beta_{1}\right)\right]=\frac{d}{d x}\left\{E_{y}^{\mathrm{TE}}\left(x,-\beta_{2}\right) H_{z}^{\mathrm{TE}}\left(x, \beta_{1}\right)\right\} \exp \left\{-i k_{0}\left(\beta_{1}-\beta_{2}\right) z\right\}+ \\
& +i k_{0}\left(\beta_{1}-\beta_{2}\right) E_{y}^{\mathrm{TE}}\left(x,-\beta_{2}\right) H_{x}^{\mathrm{TE}}\left(x, \beta_{1}\right) \exp \left\{-i k_{0}\left(\beta_{1}-\beta_{2}\right) z\right\} .
\end{aligned}
$$


Similarly,

$$
\begin{aligned}
\operatorname{div} & {\left[\vec{E}_{1}^{\mathrm{TM}}\left(\beta_{1}\right) \times \vec{H}_{2}^{\mathrm{TM}}\left(\beta_{2}\right)\right]=-\frac{d}{d x}\left\{E_{z}^{\mathrm{TM}}\left(x,-\beta_{21}\right) H_{y}^{\mathrm{TM}}\left(x, \beta_{1}\right)\right\} \exp \left\{-i k_{0}\left(\beta_{1}-\beta_{2}\right) z\right\}-} \\
- & i k_{0}\left(\beta_{1}-\beta_{2}\right) E_{x}^{\mathrm{TM}}\left(x,-\beta_{2}\right) H_{y}^{\mathrm{TM}}\left(x, \beta_{1}\right) \exp \left\{-i k_{0}\left(\beta_{1}-\beta_{2}\right) z\right\}, \\
& \operatorname{div}\left[\vec{E}_{2}^{\mathrm{TM}}\left(\beta_{2}\right) \times \vec{H}_{1}^{\mathrm{TM}}\left(\beta_{1}\right)\right]=-\frac{d}{d x}\left\{E_{z}^{\mathrm{TM}}\left(x,-\beta_{2}\right) H_{y}^{\mathrm{TM}}\left(x, \beta_{1}\right)\right\} \exp \left\{-i k_{0}\left(\beta_{1}-\beta_{2}\right) z\right\}- \\
& -i k_{0}\left(\beta_{1}-\beta_{2}\right) E_{x}^{\mathrm{TM}}\left(x,-\beta_{2}\right) H_{y}^{\mathrm{TM}}\left(x, \beta_{1}\right) \exp \left\{-i k_{0}\left(\beta_{1}-\beta_{2}\right) z\right\} .
\end{aligned}
$$

For the guided waveguide modes the integral of the first terms in (27)-(30) equals zero. As a consequence, according to (19), (20), it results that the expressions of the scalar products calculated in the two ways described above coincide.

\section{Discussion}

The calculations of the last section can be repeated for the adiabatic waveguide modes in order to get the desired scalar product in the space of tangential vector fields of the adiabatic waveguide modes. The scalar product (22)-(23) can also be used to describe the diffraction of the waveguide modes at the interconnection of two regular planar waveguides. The obtained results are to be compared with similar results obtained from the boundary Maxwell conditions for the tangential (with respect to the plane of waveguides interconnection) components of the electromagnetic field.

The resulting scalar product may be used to solve the problem of reflection and transmission of guided modes at the interconnection of planar waveguides. The described procedure enables modeling the transition of "horn" type between two planar waveguides using the built sesquilinear form.

\section{Acknowledgements}

The work is partially supported by RFBR grants No's 13-01-00595, 14-01-00628 and 15-07-08795.

\section{References}

[1] L.A. Sevastianov, A.A. Egorov, A.L. Sevastianov, Physics of Atomic Nuclei 76, 2, 224-239 (2013)

[2] A.L. Sevastianov, L.A. Sevastianov, A.A. Tiutiunnik, Matem. Mod. 27, 7, 103-110 (2015)

[3] R. E. Collin, Field Theory of Guided Waves, 2nd ed. (Wiley, New York, 1991)

[4] N.N. Fedorov, Fundamentals of Electrodynamics (Higher School, Moscow, 1980) 399 pp. [in Russian]

[5] R. F. Oulton, D. F. P. Pile, Y. Liu, and X. Zhang, Phys. Rev. B 76, 035408 (2007)

[6] M.N. Gevorkyan, D.S. Kulyabov, K.P. Lovetskiy, A.L. Sevastianov, L.A. Sevastianov, Mathematical Modelling and Geometry 3, 1, 43-63 (2015)

[7] V.V. Shevchenko, Phys. Usp. 50, 287-292 (2007) 